\title{
Combination Antibiotic Treatment of Serious Methicillin-Resistant Staphylococcus aureus Infections
}

\author{
J. S. Davis, MBBS(Hons), DTM\&H, FRACP, PhD ${ }^{1,2}$ S. Van Hal, MBChB, FRACP, FRCPA, PhD ${ }^{3,4}$
}

S. Y. C. Tong, MBBS(Hons), FRACP, PhD ${ }^{1,5}$

${ }^{1}$ Global and Tropical Health Division, Menzies School of Health Research, Darwin, Northern Territory, Australia

2 Department of Infectious Diseases, John Hunter Hospital, Newcastle, New South Wales, Australia

3 Department of Microbiology and Infectious Diseases, Royal Prince Alfred Hospital, Sydney, Australia

${ }^{4}$ Antibiotic Resistance and Mobile Elements Group, University of Western Sydney, Sydney, Australia

${ }^{5}$ Department of Infectious Diseases, Royal Darwin Hospital, Darwin, Northern Territory, Australia

Address for correspondence J.S. Davis, MBBS(Hons), DTM\&H, FRACP, $\mathrm{PhD}$, Menzies School of Health Research, Rocklands Drive, Tiwi, NT 0810, Australia (e-mail: Joshua.Davis@menzies.edu.au).

Semin Respir Crit Care Med 2015;36:3-16.

Methicillin-resistant Staphylococcus aureus (MRSA) is the most common antibiotic-resistant human pathogen, with an estimated 1,650 cases of blood stream infection and 500 deaths annually in Australia, ${ }^{1} 11,000$ deaths annually in the United States, ${ }^{2}$ and an annual excess cost to Europe's health care system of $€ 380 \mathrm{~m} .{ }^{3}$ MRSA bacteremia has a mortality of 20 to $30 \%$, exceeding that of methicillin-sensitive S. aureus (MSSA) at least in part due to the shortcomings of vanco- mycin, the standard therapy for MRSA bacteremia. Vancomycin still remains the principal agent of choice in the treatment of MRSA. ${ }^{4}$ However, multiple shortcomings of glycopeptide monotherapy have been recognized and include poor tissue penetration, slow bactericidal effect, and emergence of resistance during therapy. Combination therapy may theoretically overcome some of these deficiencies.
Issue Theme Antimicrobial Resistance: Management of Superbugs; Guest Editor, David L. Paterson, MBBS, PhD, FRACP, FRCPA
Copyright (c) 2015 by Thieme Medical Publishers, Inc., 333 Seventh Avenue, New York, NY 10001, USA. Tel: +1(212) 584-4662.
DOI http://dx.doi.org/ 10.1055/s-0034-1396906. ISSN 1069-3424. 
Consequently, when exploring therapeutic combinations, the addition of a second agent is usually directed to address one or more of the above vancomycin deficiencies: to broaden the spectrum of activity (to include resistant isolates such as heteroresistant vancomycin intermediate S. aureus [hVISA]) and increase the bacteriocidal activity of vancomycin through synergy. Other potential benefits of adding antibiotics include enhancing treatment by providing better tissue or biofilm penetration and reducing toxin production especially in toxin-mediated infections. The alternative to combination therapy is to find a better agent than vancomycin. Several agents with useful activity against MRSA have reached the market in recent years, including daptomycin, linezolid, tigecycline, and quinupristin/dalfopristin. However, none of these agents has been shown to be superior to vancomycin, with clinical trials showing daptomycin is noninferior for $S$. aureus bacteremia (SAB) and endocarditis ${ }^{5}$ and linezolid noninferior for $S$. aureus catheter-related blood stream infection, ${ }^{6}$ and although linezolid achieved improved clinical cure for MRSA pneumonia, it did not result in reduced mortality. ${ }^{7}$ Hence, combination therapy is an attractive possibility for improving outcomes from severe MRSA infections.

Most data on combination therapy come from in vitro experiments. These have three possible outcomes: synergy (increased bacteriocidal activity in excess of the additive actions of the two drugs), antagonism, and indifference. The most common in vitro methods used to measure synergy (in increasing order of complexity) are agar diffusion, checkerboard testing, time-kill curves, and simulated pharmacodynamic (PD) models. Agar diffusion methods use combinations of antibiotics either as antibiotic discs or Etest strips (Biomerieux, Paris, France; paper strips of graduated antibiotic concentration), allowing them to diffuse through the agar, and qualitatively examines bacterial growth in different zones of the agar. For example, two Etest strips (one for each antibiotic) can be placed at right angles on the agar plate, and bacterial growth examined near the intersection (where the combination concentration is highest) compared with the outer quadrants. Checkerboard testing uses multiple different dilutions of antibiotic combinations set out on an agar plate as a grid, ranging from below to above the minimum inhibitory concentration (MIC) for each agent. This allows a quantitative estimate of synergy and results are reported as a fractional inhibitory concentration (FIC). An FIC of $\leq 0.5$ indicates synergy, $\geq 2.0$ antagonism, and an FIC between these values indicates indifference. Time-kill methodology is dynamic rather than examining synergy at a single time point and thus is more likely to correlate with clinical use. Bacteria are cultured in liquid media in the presence of various concentrations of either single or combination antibiotics, and the speed of bacterial killing is quantified over time. In time-kill studies, synergy is defined as a reduction in $\geq 2 \log$ colony forming units (cfu) of bacteria/mL compared with the $\mathrm{cfu} / \mathrm{mL}$ of the most potent single drug. Finally, in vitro pharmacokinetic (PK)/PD models attempt to simulate antibiotic dosing within a human or animal host. An example is the fibrin clot model, where an ex vivo human blood clot is seeded with bacteria and exposed to dynamic concentrations of antibiotic, simulating a usual dosing interval.

\section{Combinations of Two Primarily Active Agents}

\section{Vancomycin and Linezolid}

Linezolid is an oxazolidinone, a new class of antibiotic with a mechanism of action directed at the early steps of protein synthesis. Its introduction into clinical medicine heralded the first new antibiotic with activity against MRSA since the introduction of vancomycin.

Linezolid and vancomycin combination therapy are reported to demonstrate indifference using checkerboard assays, ${ }^{8}$ but synergy with time-kill assays. ${ }^{9}$ Other studies, using similar methodology, were unable to demonstrate synergy but observed antagonism ${ }^{10,11}$ or indifference. ${ }^{12}$ Subsequent animal data have similarly been conflicting with an experimental rabbit endocarditis model, ${ }^{13}$ observing some effect with the addition of linezolid, while a rat MSSA osteomyelitis model ${ }^{14}$ showed indifference with no additional sterilization or cure rates compared with vancomycin alone. The inconsistency of these results suggests that there are a variety of mechanisms involved in determining antibiotic interactions, which may include the infecting bacterial strain, infection site, and host responses. Thus, this combination should be used with caution as the described antagonism may lead to suboptimal outcomes.

Nevertheless, this combination is still considered in toxinmediated infections, as subinhibitory concentrations of linezolid inhibit $S$. aureus toxin production. ${ }^{15,16}$ This modulation of virulence factors through reduced pathogen toxin synthesis may theoretically attenuate disease and influence outcomes. This hypothesis has not been shown in vivo to be effective, with no animal model data available. Although a single case report has been published showing the effectiveness of linezolid in the treatment of MSSA toxic shock syndrome, ${ }^{17}$ no comparative data of the combination of linezolid with vancomycin in toxin-mediated clinical syndromes has been published.

\section{Vancomycin and Daptomycin}

Daptomycin is a lipopeptide, which acts on the cell membrane through a complex process resulting in cell membrane depolarization and permeabilization, ion leakage, and ultimately cell death. ${ }^{18}$ There is a paucity of in vitro and in vivo data for this combination due to concerns about the relationship between reduced vancomycin susceptibility and daptomycin nonsusceptibility. ${ }^{19}$ This cross-resistance was first documented in vitro when a collection of VISA isolates underwent susceptibility testing and $80 \%$ were found to be daptomycin nonsusceptible, despite these isolates not having been exposed to daptomycin. ${ }^{20}$ This association was later confirmed by serial passage studies, with all S. aureus isolates developing daptomycin nonsusceptibility in the presence of vancomycin. ${ }^{21}$ Subsequently, several clinical cases have described the same phenomenon. ${ }^{22}$ Although the precise mechanism remains unclear, experts speculate that increased cell wall thickness, as occurs in VISA, may also prevent daptomycin penetration to its site of action. ${ }^{23,24}$ 
Nevertheless, Tsuji and Rybak performed Etest synergy testing and time-kill experiments on one vancomycin heteroresistant and one vancomycin intermediate clinical S. aureus isolate. There was moderate agreement between the two methodologies with vancomycin/daptomycin combination showing either indifference or an additive effect (but not synergy). ${ }^{25}$ This combination, albeit with the addition of rifampin, has been used in one published report of two cases. Both patients had orthopedic infections that relapsed after initial vancomycin/rifampin therapy and were cured with several weeks of triple therapy. ${ }^{26}$ As both patients also underwent surgical debridement and prosthesis removal, the effectiveness of daptomycin/vancomycin remains unknown, but based on the above concerns, this combination is unlikely to be successful in most cases.

\section{Vancomycin and Tetracyclines including Tigecycline}

hVISA was first isolated in Japan in the $1990 \mathrm{~s} .{ }^{27}$ Given the lack of treatment options against hVISA, combination therapy was studied. Time-kill experiments using the Mu3 strain, the first recognized hVISA isolate, revealed antagonism when using minocycline/vancomycin in combination. The authors went on to examine possible bacterial changes that may result in this antagonism and found that cell wall thickening did not play a major role.

Tigecycline is a semisynthetic derivative of minocycline and is the first glycylcyline antibiotic licensed for clinical use. ${ }^{28}$ It offered great promise as a new agent with a broad spectrum of activity against gram-negative and -positive bacteria, including multiresistant organisms. However, postmarketing experience has resulted in more selective indications, as it is associated with increased mortality when used in certain clinical settings. ${ }^{29}$ Nevertheless, given its spectrum of activity, using it in combination with vancomycin remains an attractive option. Mercier et al performed time-kill studies and found vancomycin and tigecycline to be indifferent when used with four MRSA (three of which were VISA) isolates. ${ }^{30}$ Petersen et al obtained similar results using checkerboard and time-kill experiments on $10 \mathrm{~S}$. aureus isolates. ${ }^{31}$ There have been few experimental animal studies evaluating tigecycline combinations. ${ }^{28}$ A rabbit osteomyelitis model found no difference between combination therapy compared with vancomycin or tigecycline monotherapy. ${ }^{32}$ Similarly, no difference was observed in bactericidal activity in a biofilm model for this combination. ${ }^{33}$

The main conclusion from the available data is that there is no benefit with tetracycline and vancomycin combinations. Unless tigecycline is required for a coexistent multiresistant gram-negative infection, combination with vancomycin is not recommended for the treatment of MRSA infections.

\section{Daptomycin and Tigecycline}

Time-kill and Etest experiments demonstrated an indifferent effect when using this combination. ${ }^{25}$ These results are in contrast to a subsequent study, which showed this combination to be synergistic based on time-kill studies and checkerboard titration assays using 10 S. aureus isolates. ${ }^{34}$ To corroborate their results, the authors went on to perform an animal surgical site infection model. Tigecycline/daptomycin still showed synergy. ${ }^{34}$
No clinical data are available. The role of tigecycline is likely to be limited as discussed previously and thus further studies are unlikely to occur with this combination.

\section{Daptomycin and Linezolid}

The impact of biofilm-associated infection remains significant especially in light of the aging population and increased joint replacements undertaken. Linezolid/daptomycin combination was studied by Parra-Ruiz et al in their validated in vitro PK/PD biofilm formation model. ${ }^{35}$ This study observed that linezolid/daptomycin combination therapy showed better efficacy than either agent alone and confirmed the results of one previous study using a simulated endocardial vegetation model. ${ }^{36}$ Although a single published case report showed clinical benefit, this occurred in the setting of triple therapy with daptomycin, linezolid, and rifampin. ${ }^{37}$ Thus, the benefit of this combination is unclear especially as checkerboard and time-kill experiments showed antagonism.

\section{Vancomycin and Quinupristin-Dalfopristin}

Quinupristin-dalfopristin is an injectable streptogramin antibiotic with in vitro activity against MRSA. The combination of quinupristin-dalfopristin with vancomycin has resulted in mixed results ${ }^{38}$ with studies demonstrating both antagonism $^{39}$ and synergy. ${ }^{40-43}$ There remain very limited published clinical data to guide the use of this combination. ${ }^{44,45}$ Given that quinupristin-dalfopristin is not recommended for MRSA bacteremia due to reports of treatment failures and emergent resistance, and there are no published original studies since 2002 on the combination of quinupristindalfopristin with vancomycin, it is unlikely to be further advanced as a clinical treatment option.

\section{B-Lactam Combination Therapy}

Empirical therapy for SAB often includes both vancomycin and antistaphylococcal penicillin such as nafcillin. This is not only because incorrect initial empiric therapy for MRSA bacteremia is associated with a twofold increase in mortality ${ }^{46}$ but also because vancomycin monotherapy for MSSA infections is associated with higher rates of hospitalization, ${ }^{47}$ treatment failure, ${ }^{48}$ and mortality ${ }^{49,50}$ compared with $\beta$-lactam therapy (e.g., with nafcillin or cefazolin). Given the increasing prevalence of community-acquired MRSA infection, ${ }^{51-53}$ B-lactam combination therapy is often used in patients with positive blood cultures where the Gram stain shows clustered grampositive cocci for the first 24 to 48 hours of therapy, but before identification and susceptibility profile of the organism has been determined. Hence a potentially synergistic combination is unwittingly being increasingly used in the subset that turn out to have MRSA infection. Considering the very definition of MRSA is that it is resistant to antistaphylococcal penicillins, it is counter-intuitive to hypothesize that $\beta$-lactams might have any benefit when added to standard therapy for MRSA infections. However, unexpected synergy between $\beta$-lactams and both vancomycin and daptomycin has been found to occur in vitro, initially only in VISA and hVISA strains, and then more broadly in MRSA. 


\section{Vancomycin/ $\beta$-Lactam Combinations}

\section{In Vitro Studies}

At least 16 in vitro studies have explored synergy between vancomycin and $\beta$-lactams against MRSA isolates, ${ }^{54-69}$ all but one of which found evidence of synergy in some or all of the tested strains (-Table 1 ). These studies varied in their methodology (checkerboard synergy testing or time-kill curves), types of strains tested (MRSA vs. hVISA vs. VISA) and the $\beta$-lactams used, but a consistent finding across nearly all the studies was synergistic bacterial killing in most but not all strains tested. There are no consistent characteristics in these reports in the strains where synergy was not demonstrated. However, there was a general tendency across these studies (and within some studies ${ }^{56,66}$ ) to an increasing degree of synergy with increasing vancomycin MICs. The one study that did not demonstrate synergy did not actually include any MRSA isolates. In this study, Joukhadar et al tested 10 clinical isolates of methicillin sensitive $S$. aureus and found evidence of neither synergy nor antagonism in any strain, both using fixed drug concentrations, and in a dynamic model simulating clinical dosing. ${ }^{62}$

\section{Animal Studies}

The few studies that have assessed combinations of vancomycin with $\beta$-lactams in animal models have all found evidence of synergy. ${ }^{56,61,65}$ Climo et al found faster sterilization of infection with vancomycin plus nafcillin in MRSA rabbit endocarditis and renal abscess models. ${ }^{56}$ Ribes et al tested various combinations of linezolid, vancomycin, and imipenem in a murine peritonitis VISA model using time-kill curves, and found faster bacterial killing with vancomycin plus imipenem compared with vancomycin alone, in both strains tested. ${ }^{61}$ Finally, Fernandez et al investigated the anti-MRSA cephalosporin ceftobiprole against an MRSA and a VISA strain in a rat endocarditis model. They found good activity of ceftobiprole against both strains in terms of sterilizing vegetations and preventing mortality; the combination of vancomycin plus ceftobiprole led to faster killing on time-kill curves, but similar rates of mortality and of sterilization of vegetations compared with ceftobiprole alone. ${ }^{65}$

\section{Human Studies}

There are currently no published prospective controlled trials of vancomycin/ $\beta$-lactam combination therapy in patients with MRSA bacteremia, but one observational study has recently been published. ${ }^{67}$ In this single-center retrospective cohort study, Dilworth et al described the outcomes of 50 patients with MRSA bacteremia who received combination therapy with vancomycin and at least 24 hours of $\beta$-lactam (at their clinicians' discretion), and compared them with 30 patients treated at the same hospital, during the same time period with vancomycin alone. They found a higher rate of microbiological eradication in the combination therapy group ( 96 vs. $80 \%, p=0.02$ ), which persisted on a multivariate model attempting to control for potential confounders (adjusted odds ratio for achieving microbiological eradication in the combination group $=11.24, p=0.01$ ) .

\section{Daptomycin/ $\beta$-Lactam Combinations}

\section{In Vitro Studies}

At least 10 in vitro studies have examined the combination of daptomycin with various $\beta$-lactams against MRSA and VISA strains (- Table 2). ${ }^{25,70-79}$ The findings of these studies are remarkably similar to the vancomycin/ $\beta$-lactam synergy articles cited earlier: synergy for most but not all strains tested, and an increasing degree of synergy with increasing MICs to both vancomycin and daptomycin. No studies have found evidence of antagonism with this combination.

\section{Animal Studies}

A recently published animal study mirrored the findings of the in vitro studies. Garrigós et al used a rat tissue cage model of MRSA infection to study the combination of daptomycin with cloxacillin, and found superior cure rates with the combination than with daptomycin alone. ${ }^{80}$

\section{Human Studies}

As for the vancomycin/ $\beta$-lactam combination, there are no clinical trials of daptomycin with $\beta$-lactams either published or in trials registries. However, limited observational data suggest this combination may be effective, particularly MRSA with poor response to daptomycin. In a case series of seven patients with persistent MRSA bacteremia for more than 1 week despite high-dose daptomycin, all cleared their bacteremia within 48 hours once nafcillin or oxacillin was added to their therapy. ${ }^{81}$ In a second case series of 22 patients with persistent MRSA bacteremia despite daptomycin for a median of 10 days, the addition of ceftaroline lead to clearance of bacteremia in all cases, in a median of 2 days. ${ }^{82}$

\section{Summary}

Although the studies on $\beta$-lactam combination therapy are heterogeneous, there are some consistent findings (with either vancomycin or daptomycin as the companion agent): adding a $\beta$-lactam leads to synergistic bacterial killing in the majority of strains tested and in all animal models tested. The most consistent data come from more resistant strains and from antistaphylococcal penicillins or ceftaroline rather than other $\beta$-lactams. $\beta$-lactam combination therapy (with either vancomycin or daptomycin) is not recommended in Infectious Diseases Society of America (IDSA) or other guidelines at this stage, but the emerging data are intriguing, and at least one phase $2 \mathrm{~b}$ randomized controlled trial (RCT) of this strategy is underway (Australia and New Zealand Clinical Trials Registry number ACTRN12610000940077). A key question that emerges from these data is: what is the mechanism of the observed synergy? The mechanisms have not been entirely elucidated, but are becoming clearer over time. Increasing vancomycin resistance in S. aureus is paradoxically associated with decreasing MICs to oxacillin, and this so-called "see-saw effect" due to alteration of the MecA gene in some strains of VISA and vancomycin resistant Staphylococcus aureus (VRSA), ${ }^{84,85}$ and possibly to other structural changes in penicillin-binding proteins. $\beta$-lactams have been shown to enhance binding of daptomycin to the bacterial cell wall. ${ }^{79}$ Finally, Sakoulas et al recently 


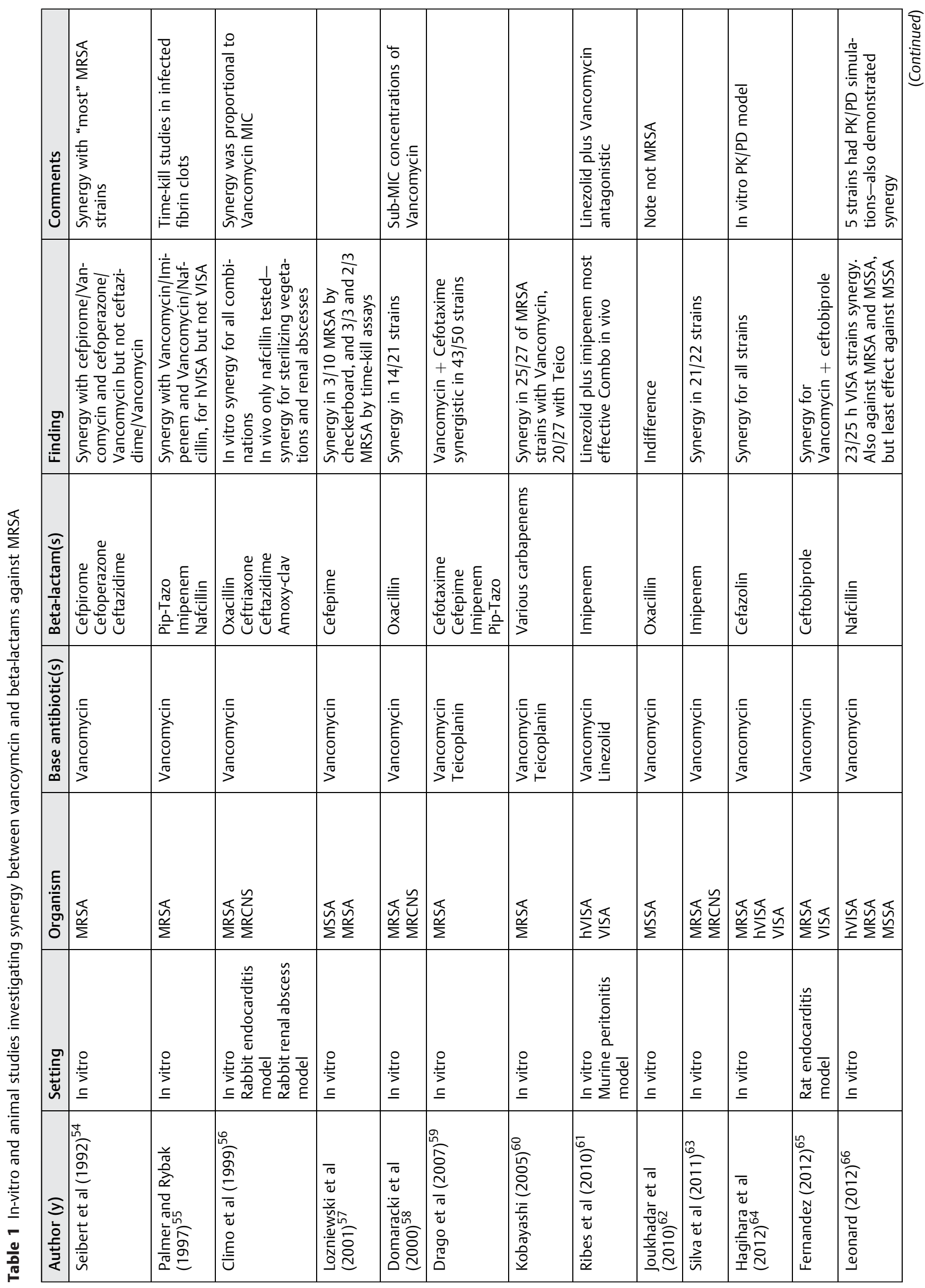




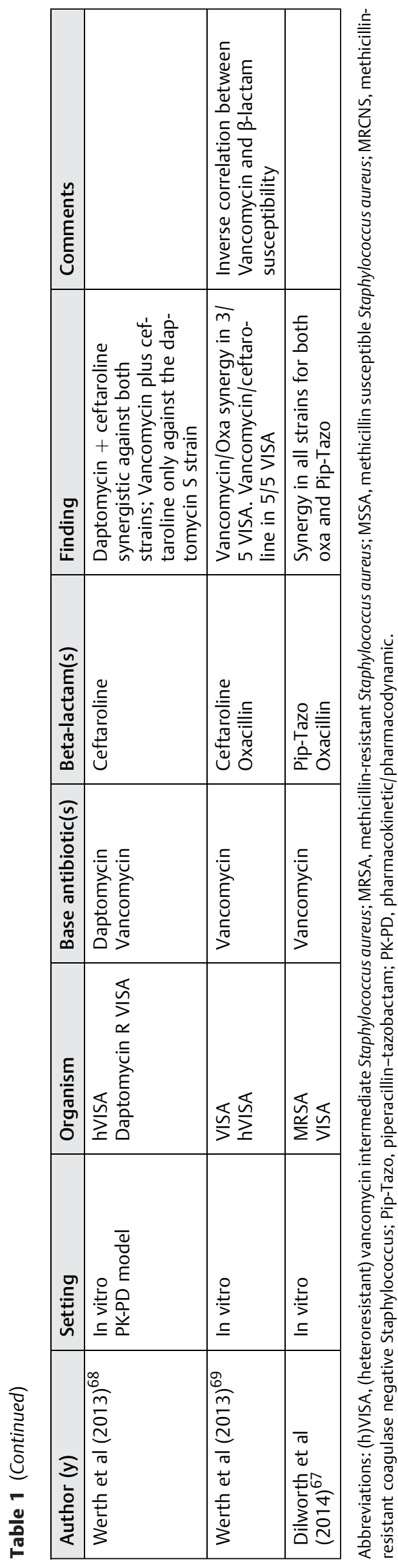

reported exciting data derived from ex vivo study of human blood which adds another potential advantage for the use of $\beta$-lactams for MRSA-they lead to increased activity of innate host defense peptides such as cathelicidin LL- $37,{ }^{86}$ which in turn allows more efficient bacterial killing.

\section{Other Combination Therapy}

\section{Rifampin}

\section{Vancomycin/Rifampin}

Rifampin is attractive as an adjunctive agent as it is bactericid$\mathrm{al},{ }^{87}$ has activity against cells in stationary growth phase, ${ }^{88}$ and is better able to penetrate cells, ${ }^{89,90}$ tissues, and biofilms ${ }^{91,92}$ than vancomycin. ${ }^{93,94}$ However, most in vitro studies demonstrate either antagonism or indifference for the combination of rifampin with vancomycin. ${ }^{25,87,95-102}$ There is some in vitro evidence of synergy in the presence of biofilm production ${ }^{33,103}$ and in animal models of osteomyelitis ${ }^{96,104}$ or foreign body infections. ${ }^{101,105,106}$ Bayer and Lam found in a rabbit MRSA endocarditis model that the combination of vancomycin and rifampin improved valvular sterilization and overall cure. ${ }^{107}$ However, these findings have not been replicated in other animal endocarditis models. ${ }^{108,109} \mathrm{~A}$ systematic review of in vitro and animal experiments specifically addressing the benefit of adding rifampin to other antibiotics for S. aureus infections concluded that the effect of rifampin therapy was often inconsistent and method dependent. ${ }^{92}$

Clinical studies to date have not provided evidence in support of the combination of vancomycin with rifampin for severe MRSA infections. Levine et al randomized 42 patients with MRSA endocarditis to vancomycin or vancomycin plus rifampin. ${ }^{110}$ The median duration of bacteremia was longer in the combination arm (9 vs. 7 days) and rates of treatment failure were similar. Similarly, Riedel et al determined in a retrospective cohort of 84 patients with $S$. aureus native valve infective endocarditis (IE) that the addition of rifampin was associated with longer duration of bacteremia and increased mortality compared with controls. ${ }^{111}$ There was also a higher incidence of hepatotoxicity and significant drug-drug interactions for patients receiving rifampin. ${ }^{112}$ Jung et al conducted a RCT in patients with MRSA nosocomial pneumonia. ${ }^{112}$ Clinical cure at day 14 was achieved in 54\% (22 of 41 ) of patients in the vancomycin plus rifampin arm compared with $31 \%$ (13 of 42 ) in the vancomycin alone $\operatorname{arm}(p=0.047)$. Although these data are promising, the study was unblended and single center, and vancomycin dosing (1 g q12h) was lower than current recommendations. Based on the Levine et $\mathrm{al}^{110}$ and Riedel et al ${ }^{111}$ studies, the IDSA MRSA treatment guidelines recommend against the addition of rifampin to vancomycin for MRSA bacteremia or native valve IE (level A-I; good evidence, at least one properly RCT). ${ }^{4}$

Nonetheless, Thwaites et al have cogently argued that equipoise exists for the addition of rifampin to standard therapy for $S A B$ and a RCT comparing standard therapy to standard therapy plus adjunctive rifampin for $S A B$ in the United Kingdom is currently being conducted (the adjunctive rifampicin to reduce early mortality from Staphylococcus 


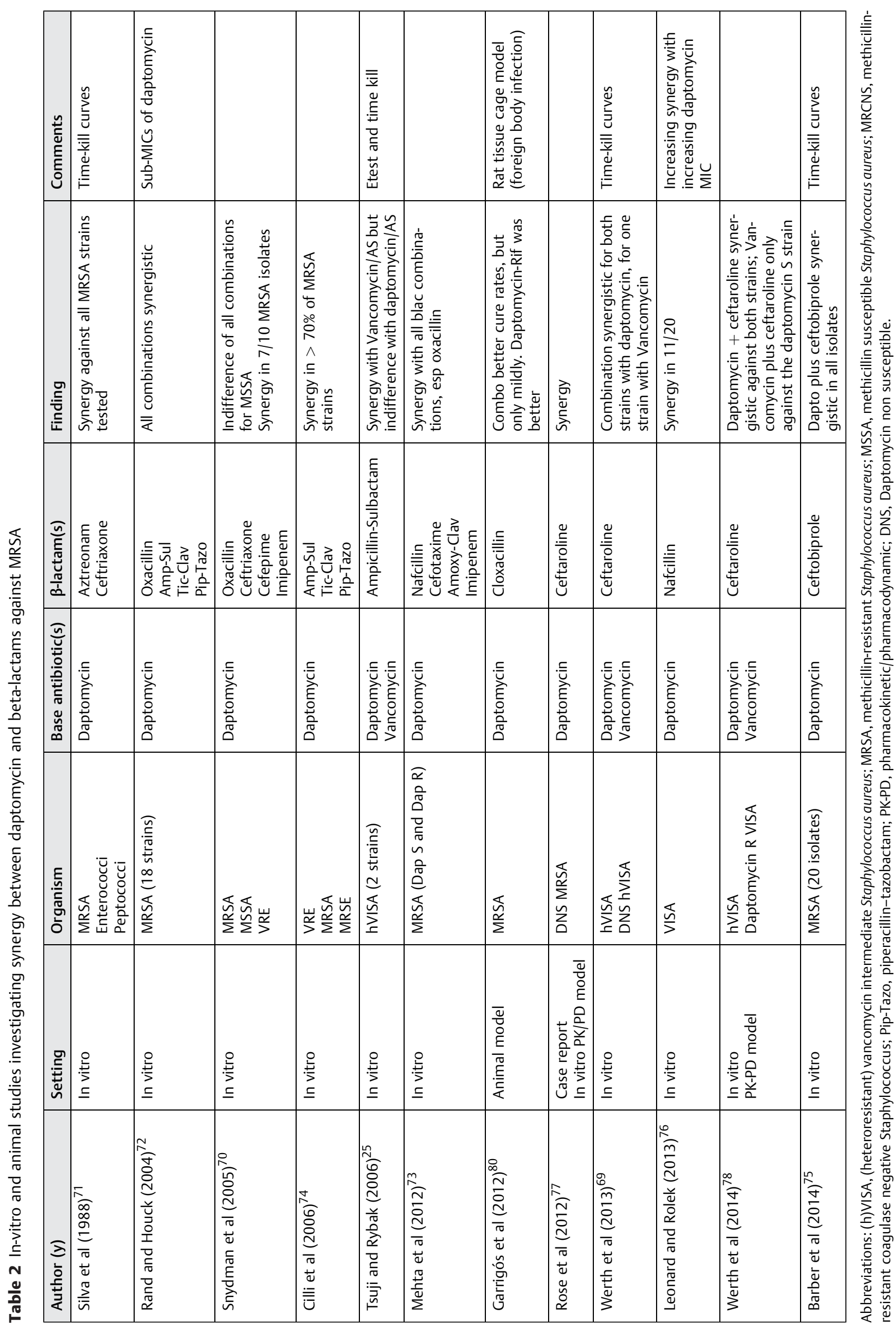


aureus bacteraemia [ARREST] study). ${ }^{113}$ In a review of four previously published RCTs, ${ }^{110,112,114,115}$ Thwaites et al determined that adjunctive rifampin for serious staphylococcal infections was associated with a reduction in infection-related deaths by $55 \%(p=0.02){ }^{113}$ Notably, two of these studies principally focused on MSSA infections treated with oxacillin, ${ }^{114,115}$ and the combined findings in this systematic review probably do not apply to MRSA and vancomycin therapy. In the ARREST trial, MRSA bacteremia is not a prespecified subgroup. Thus, despite this being the largest planned RCT for SAB to date, the study may be underpowered to make specific conclusions regarding the MRSA subgroup, particularly as reductions in numbers of MRSA bacteremia in the United Kingdom may result in MRSA bacteremia being a minority of infections. ${ }^{116}$

There is stronger but still inconclusive evidence for the use of adjunctive rifampin for prosthetic joint infections (PJIs), where biofilm assumes a critical importance. For example, Peel et al ${ }^{117}$ and Aboltins et al ${ }^{118}$ have reported on successful outcomes with debridement and retention of carefully selected patients with PJI prescribed prolonged courses of rifampin and fusidic acid. However, these studies suffer from their retrospective and observational nature, a limited number of patients with MRSA $(n=39)$, and notably MRSA infection (compared with coagulase negative staphylococci) remained an independent risk factor for treatment failure. ${ }^{117}$ The combination of a fluoroquinolone with rifampin has also been demonstrated to be effective in treating selected PJIs with a debridement and retention approach in both a small RCT $^{119}$ and several retrospective cohort studies. ${ }^{120-122}$ However, the RCT reported by Zimmerli et $\mathrm{al}^{119}$ only included $15 \mathrm{PJI}$ patients, included a relatively ineffective control arm (ciprofloxacin monotherapy), and when reanalyzed by intention to treat found no significant difference between the rifampicin and nonrifampicin containing arms. Somewhat controversially, ${ }^{123}$ based largely on this single RCT, the 2013 IDSA guidelines recommend that rifampin be added to initial parenteral therapy for MRSA PJI, followed by prolonged combination oral therapy with rifampin with a companion agent such as a fluoroquinolone. $^{124}$

\section{Daptomycin/Rifampin}

In vitro and animal studies demonstrate an overall pattern of either antagonism or indifference with the addition of rifampin to daptomycin. For example, in an in vitro IE model, the combination of daptomycin with either rifampin or gentamicin antagonized or delayed the bactericidal activity of daptomycin alone. ${ }^{125}$ Similarly, in time-kill experiments and a rabbit endocarditis model, the combination of daptomycin and either rifampin or gentamicin demonstrated no enhancement of the effectiveness of daptomycin against MRSA compared with daptomycin alone. ${ }^{126}$ There are currently only case reports or small case series of clinical studies involving the combination of daptomycin and rifampin. ${ }^{127-129}$

\section{Gentamicin}

Vancomycin/Gentamicin

Several studies have demonstrated a consistent in vitro synergism between aminoglycosides and vancomycin. ${ }^{130,131}$
However, clinical studies do not support the addition of an aminoglycoside to vancomycin. In a retrospective evaluation of 87 patients with persistent SAB, 48 of whom had MRSA infection, those treated with an aminoglycoside had lower incidence of recurrence within 6 months, but there was no significant association with mortality or other outcomes. ${ }^{132}$ In analyzing data from the daptomycin registrational $\mathrm{RCT},{ }^{5}$ Cosgrove et al found that $27 / 122$ (22\%) of patients who received initial low-dose gentamicin therapy (in combination with either nafcillin or vancomycin) experienced a clinically significant decline in renal function, compared with $8 / 100$ $(8 \%)$ of those who did not receive gentamicin. ${ }^{133}$ Based on these clinical studies, the IDSA recommends that gentamicin should not be added to vancomycin for the treatment of MRSA bacteremia or native valve endocarditis. ${ }^{4}$

\section{Daptomycin/Gentamicin}

The combination of daptomycin with gentamicin has been tested in vitro with varying results; synergy has been demonstrated in some studies, ${ }^{70,134-136}$ but not in others. ${ }^{25,125,126,137-140}$ Unfortunately, a RCT comparing daptomycin to daptomycin combined with gentamicin was terminated early after recruiting only 24 patients (Clinical trials NCT00638157). Thus, the combination of daptomycin with gentamicin cannot be recommended at this stage.

\section{Other Combinations}

Daptomycin-nonsusceptible S. aureus (DNS) not infrequently emerges during daptomycin therapy. ${ }^{5,141}$ Among agents tested in combination with daptomycin, trimethoprim-sulfamethoxazole has shown promise in a PK/PD model for the treatment of DNS. ${ }^{36,142}$ Although clinical experience is currently limited ${ }^{143,144}$ for DNS infections that are refractory to standard treatment, the combination with trimethoprimsulfamethoxazole should be considered.

In vitro studies have determined that subinhibitory concentrations of clindamycin, linezolid, and rifampin can block production of toxins such as Panton-Valentine leukocidin and $\alpha$-toxin by S. aureus. ${ }^{145-147}$ Clinical experience of the use of these agents in severe toxin-mediated staphylococcal infections (e.g., toxic shock syndrome or necrotizing pneumonia) is limited. ${ }^{148}$ Two retrospective studies suggest that there may be a clinical benefit for suppression of toxins in such cases. ${ }^{149,150}$ Treatment guidelines from the United Kingdom and France recommend that antitoxin therapy be instituted where toxin-mediated staphylococcal disease is suspected or apparent ${ }^{151,152}$ and in the absence of robust evidence for the treatment of these life-threatening infections, these recommendations are clearly sensible.

Studies of combination therapy for MRSA involving novel antibiotics are also beginning to emerge. In a small number of clinical MRSA isolates tested in vitro (five VISA and five hVISA), oritavancin (a novel lipoglycopeptide antibiotic) appears to be synergistic when used in combination with either gentamicin, linezolid or rifampin, ${ }^{153}$ as does telavancin (a second novel lipoglycopeptide antibiotic), when used with gentamicin, ceftriaxone, rifampin, or meropenem. ${ }^{154}$ Since only these two drugs became Food and Drug 
Administration approved in 2014, and are not approved for treatment of MRSA bacteremia, clinical experience is limited and the implications of these in vitro studies are unclear at this stage. Despite its lack of activity as a single agent against MRSA, fosfomycin appears to be synergistic with linezolid against clinical MRSA isolates in an in vitro model ${ }^{155}$ mirroring the $\beta$-lactam concept, where a seemingly inactive agent makes an important contribution when combined with an active agent.

\section{Conclusion}

Because of the limitations of vancomycin, the standard therapy for serious MRSA infections, many combinations of antibiotics have been tested, primarily in in vitro models. Unfortunately, studies of the majority of these combinations have reported mixed or negative data. However, several $\beta$-lactam antibiotics have consistently been shown to be synergistic for the majority of MRSA strains (including hVISA and daptomycin nonsusceptible strains), when combined with either vancomycin or daptomycin. Although these combinations appear promising, limited clinical data are available, and clinical trials are only just beginning to be performed. Currently, there is insufficient evidence to recommend any combination therapy for serious MRSA infections in actual patient care.

\section{References}

1 Turnidge JD, Kotsanas D, Munckhof W, et al; Australia New Zealand Cooperative on Outcomes in Staphylococcal Sepsis. Staphylococcus aureus bacteraemia: a major cause of mortality in Australia and New Zealand. Med J Aust 2009;191(7):368-373

2 Dantes R, Mu Y, Belflower R, et al; Emerging Infections ProgramActive Bacterial Core Surveillance MRSA Surveillance Investigators. National burden of invasive methicillin-resistant Staphylococcus aureus infections, United States, 2011. JAMA Intern Med 2013;173(21):1970-1978

3 Köck R, Becker K, Cookson B, et al. Methicillin-resistant Staphylococcus aureus (MRSA): burden of disease and control challenges in Europe. Euro Surveill 2010;15(41):19688

4 Liu C, Bayer A, Cosgrove SE, et al. Clinical practice guidelines by the Infectious Diseases Society of America for the treatment of methicillin-resistant Staphylococcus aureus infections in adults and children. Clin Infect Dis 2011;52:e18-55

5 Fowler VG Jr, Boucher HW, Corey GR, et al; S. aureus Endocarditis and Bacteremia Study Group. Daptomycin versus standard therapy for bacteremia and endocarditis caused by Staphylococcus aureus. N Engl J Med 2006;355(7):653-665

6 Wilcox MH, Tack KJ, Bouza E, et al. Complicated skin and skinstructure infections and catheter-related bloodstream infections: noninferiority of linezolid in a phase 3 study. Clin Infect Dis 2009; 48(2):203-212

7 Wunderink RG, Niederman MS, Kollef MH, et al. Linezolid in methicillin-resistant Staphylococcus aureus nosocomial pneumonia: a randomized, controlled study. Clin Infect Dis 2012; 54(5):621-629

8 Mulazimoglu L, Drenning SD, Yu VL. In vitro activities of two novel oxazolidinones (U100592 and U100766), a new fluoroquinolone (trovafloxacin), and dalfopristin-quinupristin against Staphylococcus aureus and Staphylococcus epidermidis. Antimicrob Agents Chemother 1996;40(10):2428-2430
9 Jacqueline C, Caillon J, Le Mabecque V, et al. In vitro activity of linezolid alone and in combination with gentamicin, vancomycin or rifampicin against methicillin-resistant Staphylococcus aureus by time-kill curve methods. J Antimicrob Chemother 2003;51(4): 857-864

10 Grohs P, Kitzis MD, Gutmann L. In vitro bactericidal activities of linezolid in combination with vancomycin, gentamicin, ciprofloxacin, fusidic acid, and rifampin against Staphylococcus aureus. Antimicrob Agents Chemother 2003;47(1):418-420

11 Singh SR, Bacon AE III, Young DC, Couch KA. In vitro 24-hour timekill studies of vancomycin and linezolid in combination versus methicillin-resistant Staphylococcus aureus. Antimicrob Agents Chemother 2009;53(10):4495-4497

12 Jones RN, Anderegg TR, Deshpande LM. AZD2563, a new oxazolidinone: bactericidal activity and synergy studies combined with gentamicin or vancomycin against staphylococci and streptococcal strains. Diagn Microbiol Infect Dis 2002;43(1):87-90

13 Chiang FY, Climo M. Efficacy of linezolid alone or in combination with vancomycin for treatment of experimental endocarditis due to methicillin-resistant Staphylococcus aureus. Antimicrob Agents Chemother 2003;47(9):3002-3004

14 Patel R, Piper KE, Rouse MS, Steckelberg JM. Linezolid therapy of Staphylococcus aureus experimental osteomyelitis. Antimicrob Agents Chemother 2000;44(12):3438-3440

15 Bernardo K, Pakulat N, Fleer S, et al. Subinhibitory concentrations of linezolid reduce Staphylococcus aureus virulence factor expression. Antimicrob Agents Chemother 2004;48(2):546-555

16 Stevens DL, Ma Y, Salmi DB, McIndoo E, Wallace RJ, Bryant AE. Impact of antibiotics on expression of virulence-associated exotoxin genes in methicillin-sensitive and methicillin-resistant Staphylococcus aureus. J Infect Dis 2007;195(2):202-211

17 Stevens DL, Wallace RJ, Hamilton SM, Bryant AE. Successful treatment of staphylococcal toxic shock syndrome with linezolid: a case report and in vitro evaluation of the production of toxic shock syndrome toxin type 1 in the presence of antibiotics. Clin Infect Dis 2006;42(5):729-730

18 Bayer AS, Schneider T, Sahl HG. Mechanisms of daptomycin resistance in Staphylococcus aureus: role of the cell membrane and cell wall. Ann N Y Acad Sci 2013;1277:139-158

19 Nadrah K, Strle F. Antibiotic Combinations with Daptomycin for Treatment of Staphylococcus aureus Infections. Chemother Res Pract 2011;2011:619321

20 Patel JB, Jevitt LA, Hageman J, McDonald LC, Tenover FC. An association between reduced susceptibility to daptomycin and reduced susceptibility to vancomycin in Staphylococcus aureus. Clin Infect Dis 2006;42(11):1652-1653

21 Sakoulas G, Alder J, Thauvin-Eliopoulos C, Moellering RC Jr, Eliopoulos GM. Induction of daptomycin heterogeneous susceptibility in Staphylococcus aureus by exposure to vancomycin. Antimicrob Agents Chemother 2006;50(4):1581-1585

22 van Hal SJ, Paterson DL, Gosbell IB. Emergence of daptomycin resistance following vancomycin-unresponsive Staphylococcus aureus bacteraemia in a daptomycin-naïve patient-a review of the literature. Eur J Clin Microbiol Infect Dis 2011;30(5):603-610

23 Moise PA, North D, Steenbergen JN, Sakoulas G. Susceptibility relationship between vancomycin and daptomycin in Staphylococcus aureus: facts and assumptions. Lancet Infect Dis 2009; 9(10):617-624

24 Cui L, Tominaga E, Neoh HM, Hiramatsu K. Correlation between Reduced Daptomycin Susceptibility and Vancomycin Resistance in Vancomycin-Intermediate Staphylococcus aureus. Antimicrob Agents Chemother 2006;50(3):1079-1082

25 Tsuji BT, Rybak MJ. Etest synergy testing of clinical isolates of Staphylococcus aureus demonstrating heterogeneous resistance to vancomycin. Diagn Microbiol Infect Dis 2006;54(1):73-77

26 Antony SJ. Combination therapy with daptomycin, vancomycin, and rifampin for recurrent, severe bone and prosthetic joint 
infections involving methicillin-resistant Staphylococcus aureus. Scand J Infect Dis 2006;38(4):293-295

27 Hiramatsu K, Aritaka N, Hanaki H, et al. Dissemination in Japanese hospitals of strains of Staphylococcus aureus heterogeneously resistant to vancomycin. Lancet 1997;350(9092):1670-1673

28 Entenza JM, Moreillon P. Tigecycline in combination with other antimicrobials: a review of in vitro, animal and case report studies. Int J Antimicrob Agents 2009;34(1):8.e1-8.e9

29 Prasad P, Sun J, Danner RL, Natanson C. Excess deaths associated with tigecycline after approval based on noninferiority trials. Clin Infect Dis 2012;54(12):1699-1709

30 Mercier RC, Kennedy C, Meadows C. Antimicrobial activity of tigecycline (GAR-936) against Enterococcus faecium and Staphylococcus aureus used alone and in combination. Pharmacotherapy 2002;22(12):1517-1523

31 Petersen PJ, Labthavikul P, Jones CH, Bradford PA. In vitro antibacterial activities of tigecycline in combination with other antimicrobial agents determined by chequerboard and timekill kinetic analysis. J Antimicrob Chemother 2006;57(3): 573-576

32 Yin LY, Lazzarini L, Li F, Stevens CM, Calhoun JH. Comparative evaluation of tigecycline and vancomycin, with and without rifampicin, in the treatment of methicillin-resistant Staphylococcus aureus experimental osteomyelitis in a rabbit model. J Antimicrob Chemother 2005;55(6):995-1002

33 Rose WE, Poppens PT. Impact of biofilm on the in vitro activity of vancomycin alone and in combination with tigecycline and rifampicin against Staphylococcus aureus. J Antimicrob Chemother 2009;63(3):485-488

34 Silvestri C, Cirioni O, Arzeni D, et al. In vitro activity and in vivo efficacy of tigecycline alone and in combination with daptomycin and rifampin against Gram-positive cocci isolated from surgical wound infection. Eur J Clin Microbiol Infect Dis 2012;31(8): 1759-1764

35 Parra-Ruiz J, Bravo-Molina A, Peña-Monje A, Hernández-Quero J. Activity of linezolid and high-dose daptomycin, alone or in combination, in an in vitro model of Staphylococcus aureus biofilm. J Antimicrob Chemother 2012;67(11):2682-2685

36 Steed ME, Vidaillac C, Rybak MJ. Novel daptomycin combinations against daptomycin-nonsusceptible methicillin-resistant Staphylococcus aureus in an in vitro model of simulated endocardial vegetations. Antimicrob Agents Chemother 2010;54(12): 5187-5192

37 Kelesidis T, Humphries R, Ward K, Lewinski MA, Yang OO. Combination therapy with daptomycin, linezolid, and rifampin as treatment option for MRSA meningitis and bacteremia. Diagn Microbiol Infect Dis 2011;71(3):286-290

38 Brown J, Freeman BB III. Combining quinupristin/dalfopristin with other agents for resistant infections. Ann Pharmacother 2004;38(4):677-685

39 Fuchs PC, Barry AL, Brown SD. Interactions of quinupristindalfopristin with eight other antibiotics as measured by timekill studies with 10 strains of Staphylococcus aureus for which quinupristin-dalfopristin alone was not bactericidal. Antimicrob Agents Chemother 2001;45(9):2662-2665

40 Pavie J, Lefort A, Zarrouk V, et al. Efficacies of quinupristindalfopristin combined with vancomycin in vitro and in experimental endocarditis due to methicillin-resistant Staphylococcus aureus in relation to cross-resistance to macrolides, lincosamides, and streptogramin B- type antibiotics. Antimicrob Agents Chemother 2002;46(9):3061-3064

41 Kang SL, Rybak MJ. In-vitro bactericidal activity of quinupristin/ dalfopristin alone and in combination against resistant strains of Enterococcus species and Staphylococcus aureus. J Antimicrob Chemother 1997;39(Suppl A ):33-39

42 Kang SL, Rybak MJ. Pharmacodynamics of RP 59500 alone and in combination with vancomycin against Staphylococcus aureus in an in vitro-infected fibrin clot model. Antimicrob Agents Chemother 1995;39(7):1505-1511

43 Allen GP, Cha R, Rybak MJ. In vitro activities of quinupristindalfopristin and cefepime, alone and in combination with various antimicrobials, against multidrug-resistant staphylococci and enterococci in an in vitro pharmacodynamic model. Antimicrob Agents Chemother 2002;46(8):2606-2612

44 Scotton PG, Rigoli R, Vaglia A. Combination of quinupristin/ dalfopristin and glycopeptide in severe methicillin-resistant staphylococcal infections failing previous glycopeptide regimens. Infection 2002;30(3):161-163

45 Sgarabotto D, Cusinato R, Narne E, et al. Synercid plus vancomycin for the treatment of severe methicillin-resistant Staphylococcus aureus and coagulase-negative staphylococci infections: evaluation of 5 cases. Scand J Infect Dis 2002;34(2):122-126

46 Paul M, Kariv G, Goldberg E, et al. Importance of appropriate empirical antibiotic therapy for methicillin-resistant Staphylococcus aureus bacteraemia. J Antimicrob Chemother 2010; 65(12):2658-2665

47 Chan KE, Warren HS, Thadhani RI, et al. Prevalence and outcomes of antimicrobial treatment for Staphylococcus aureus bacteremia in outpatients with ESRD. J Am Soc Nephrol 2012;23(9): 1551-1559

48 Chang FY, MacDonald BB, Peacock JE Jr, et al. A prospective multicenter study of Staphylococcus aureus bacteremia: incidence of endocarditis, risk factors for mortality, and clinical impact of methicillin resistance. Medicine (Baltimore) 2003; 82(5):322-332

49 Khatib R, Saeed S, Sharma M, Riederer K, Fakih MG, Johnson LB. Impact of initial antibiotic choice and delayed appropriate treatment on the outcome of Staphylococcus aureus bacteremia. Eur J Clin Microbiol Infect Dis 2006;25(3):181-185

50 Schweizer ML, Furuno JP, Harris AD, et al. Comparative effectiveness of nafcillin or cefazolin versus vancomycin in methicillinsusceptible Staphylococcus aureus bacteremia. BMC Infect Dis 2011;11:279

51 Klevens RM, Morrison MA, Nadle J, et al; Active Bacterial Core surveillance (ABCs) MRSA Investigators. Invasive methicillinresistant Staphylococcus aureus infections in the United States. JAMA 2007;298(15):1763-1771

52 Popovich KJ, Weinstein RA, Hota B. Are community-associated methicillin-resistant Staphylococcus aureus (MRSA) strains replacing traditional nosocomial MRSA strains? Clin Infect Dis 2008;46(6):787-794

53 Wisplinghoff H, Bischoff T, Tallent SM, Seifert H, Wenzel RP, Edmond MB. Nosocomial bloodstream infections in US hospitals: analysis of 24,179 cases from a prospective nationwide surveillance study. Clin Infect Dis 2004;39(3):309-317

54 Seibert G, Isert D, Klesel N, Limbert M, Markus A, Schrinner E. The in-vitro antibacterial activity of a combination of cefpirome or cefoperazone with vancomycin against enterococci and Staphylococcus aureus. J Antimicrob Chemother 1992;29(Suppl A):25-30

55 Palmer SM, Rybak MJ. An evaluation of the bactericidal activity of ampicillin/sulbactam, piperacillin/tazobactam, imipenem or nafcillin alone and in combination with vancomycin against methicillin-resistant Staphylococcus aureus (MRSA) in time-kill curves with infected fibrin clots. J Antimicrob Chemother 1997;39(4): 515-518

56 Climo MW, Patron RL, Archer GL. Combinations of vancomycin and beta-lactams are synergistic against staphylococci with reduced susceptibilities to vancomycin. Antimicrob Agents Chemother 1999;43(7):1747-1753

57 Lozniewski A, Lion C, Mory F, Weber M. In vitro synergy between cefepime and vancomycin against methicillin-susceptible and -resistant Staphylococcus aureus and Staphylococcus epidermidis. J Antimicrob Chemother 2001;47(1):83-86 
58 Domaracki BE, Evans AM, Venezia RA. Vancomycin and oxacillin synergy for methicillin-resistant staphylococci. Antimicrob Agents Chemother 2000;44(5):1394-1396

59 Drago L, De Vecchi E, Nicola L, Gismondo MR. In vitro evaluation of antibiotics' combinations for empirical therapy of suspected methicillin resistant Staphylococcus aureus severe respiratory infections. BMC Infect Dis 2007;7:111

60 Kobayashi Y. Study of the synergism between carbapenems and vancomycin or teicoplanin against MRSA, focusing on S-4661, a carbapenem newly developed in Japan. J Infect Chemother 2005; 11(5):259-261

61 Ribes S, Pachón-Ibáñez ME, Domínguez MA, et al. In vitro and in vivo activities of linezolid alone and combined with vancomycin and imipenem against Staphylococcus aureus with reduced susceptibility to glycopeptides. Eur J Clin Microbiol Infect Dis 2010;29(11):1361-1367

62 Joukhadar C, Pillai S, Wennersten C, Moellering RC Jr, Eliopoulos GM. Lack of bactericidal antagonism or synergism in vitro between oxacillin and vancomycin against methicillin-susceptible strains of Staphylococcus aureus. Antimicrob Agents Chemother 2010;54(2):773-777

63 Silva LV, Araújo MT, Santos KR, Nunes AP. Evaluation of the synergistic potential of vancomycin combined with other antimicrobial agents against methicillin-resistant Staphylococcus aureus and coagulase-negative Staphylococcus spp strains. Mem Inst Oswaldo Cruz 2011;106(1):44-50

64 Hagihara M, Wiskirchen DE, Kuti JL, Nicolau DP. In vitro pharmacodynamics of vancomycin and cefazolin alone and in combination against methicillin-resistant Staphylococcus aureus. Antimicrob Agents Chemother 2012;56(1):202-207

65 Fernandez J, Abbanat D, Shang W, et al. Synergistic activity of ceftobiprole and vancomycin in a rat model of infective endocarditis caused by methicillin-resistant and glycopeptide-intermediate Staphylococcus aureus. Antimicrob Agents Chemother 2012;56(3):1476-1484

66 Leonard SN. Synergy between vancomycin and nafcillin against Staphylococcus aureus in an in vitro pharmacokinetic/pharmacodynamic model. PLoS ONE 2012;7(7):e42103

67 Dilworth TJ, Sliwinski J, Ryan K, Dodd M, Mercier RC. Evaluation of vancomycin in combination with piperacillin-tazobactam or oxacillin against clinical methicillin-resistant Staphylococcus aureus Isolates and vancomycin-intermediate $\mathrm{S}$. aureus isolates in vitro. Antimicrob Agents Chemother 2014;58(2):1028-1033

68 Werth BJ, Steed ME, Kaatz GW, Rybak MJ. Evaluation of ceftaroline activity against heteroresistant vancomycin-intermediate Staphylococcus aureus and vancomycin-intermediate methicillin-resistant S. aureus strains in an in vitro pharmacokinetic/ pharmacodynamic model: exploring the "seesaw effect". Antimicrob Agents Chemother 2013;57(6):2664-2668

69 Werth BJ, Vidaillac C, Murray KP, et al. Novel combinations of vancomycin plus ceftaroline or oxacillin against methicillinresistant vancomycin-intermediate Staphylococcus aureus (VISA) and heterogeneous VISA. Antimicrob Agents Chemother 2013;57(5):2376-2379

70 Snydman DR, McDermott LA, Jacobus NV. Evaluation of in vitro interaction of daptomycin with gentamicin or beta-lactam antibiotics against Staphylococcus aureus and Enterococci by FIC index and timed-kill curves. J Chemother 2005;17(6): 614-621

71 Silva M, Jacobus NV, Gorbach SL. In vitro activity of LY146032 against gram-positive bacteria. Diagn Microbiol Infect Dis 1988; $9(2): 79-85$

72 Rand KH, Houck HJ. Synergy of daptomycin with oxacillin and other beta-lactams against methicillin-resistant Staphylococcus aureus. Antimicrob Agents Chemother 2004;48(8):2871-2875

73 Mehta S, Singh C, Plata KB, et al. $\beta$-Lactams increase the antibacterial activity of daptomycin against clinical methicillin-resistant Staphylococcus aureus strains and prevent selection of dapto- mycin-resistant derivatives. Antimicrob Agents Chemother 2012; 56(12):6192-6200

74 Cilli F, Aydemir S, Tunger A. In vitro activity of daptomycin alone and in combination with various antimicrobials against Grampositive cocci. J Chemother 2006;18(1):27-32

75 Barber KE, Werth BJ, Ireland CE, et al. Potent synergy of ceftobiprole plus daptomycin against multiple strains of Staphylococcus aureus with various resistance phenotypes. J Antimicrob Chemother 2014;69(11):3006-3010

76 Leonard SN, Rolek KM. Evaluation of the combination of daptomycin and nafcillin against vancomycin-intermediate Staphylococcus aureus. J Antimicrob Chemother 2013;68(3):644-647

77 Rose WE, Schulz LT, Andes D, et al. Addition of ceftaroline to daptomycin after emergence of daptomycin-nonsusceptible Staphylococcus aureus during therapy improves antibacterial activity. Antimicrob Agents Chemother 2012;56(10):5296-5302

78 Werth BJ, Barber KE, Ireland CE, Rybak MJ. Evaluation of ceftaroline, vancomycin, daptomycin, or ceftaroline plus daptomycin against daptomycin-nonsusceptible methicillin-resistant Staphylococcus aureus in an in vitro pharmacokinetic/pharmacodynamic model of simulated endocardial vegetations. Antimicrob Agents Chemother 2014;58(6):3177-3181

79 Werth BJ, Sakoulas G, Rose WE, Pogliano J, Tewhey R, Rybak MJ. Ceftaroline increases membrane binding and enhances the activity of daptomycin against daptomycin-nonsusceptible vancomycin-intermediate Staphylococcus aureus in a pharmacokinetic/ pharmacodynamic model. Antimicrob Agents Chemother 2013; 57(1):66-73

80 Garrigós C, Murillo O, Lora-Tamayo J, et al. Efficacy of daptomycin-cloxacillin combination in experimental foreign-body infection due to methicillin-resistant Staphylococcus aureus. Antimicrob Agents Chemother 2012;56(7):3806-3811

81 Dhand A, Bayer AS, Pogliano J, et al. Use of antistaphylococcal beta-lactams to increase daptomycin activity in eradicating persistent bacteremia due to methicillin-resistant Staphylococcus aureus: role of enhanced daptomycin binding. Clin Infect Dis 2011;53(2):158-163

82 Sakoulas G, Moise PA, Casapao AM, et al. Antimicrobial Salvage Therapy for Persistent Staphylococcal Bacteremia Using Daptomycin Plus Ceftaroline. Clin Ther 2014

83 Ortwine JK, Werth BJ, Sakoulas G, Rybak MJ. Reduced glycopeptide and lipopeptide susceptibility in Staphylococcus aureus and the "seesaw effect": Taking advantage of the back door left open? Drug Resist Updat 2013;16(3-5):73-79

84 Adhikari RP, Scales GC, Kobayashi K, Smith JM, Berger-Bächi B, Cook GM. Vancomycin-induced deletion of the methicillin resistance gene mecA in Staphylococcus aureus. J Antimicrob Chemother 2004;54(2):360-363

85 Sieradzki K, Wu SW, Tomasz A. Inactivation of the methicillin resistance gene mecA in vancomycin-resistant Staphylococcus aureus. Microb Drug Resist 1999;5(4):253-257

86 Sakoulas G, Okumura CY, Thienphrapa W, et al. Nafcillin enhances innate immune-mediated killing of methicillin-resistant Staphylococcus aureus. J Mol Med (Berl) 2014;92(2):139-149

87 Tuazon CU, Lin MY, Sheagren JN. In vitro activity of rifampin alone and in combination with nafcillin and Vancomycin against pathogenic strains of Staphylococcus aureus. Antimicrob Agents Chemother 1978;13(5):759-761

88 Bahl D, Miller DA, Leviton I, et al. In vitro activities of ciprofloxacin and rifampin alone and in combination against growing and nongrowing strains of methicillin-susceptible and methicillinresistant Staphylococcus aureus. Antimicrob Agents Chemother 1997;41(6):1293-1297

89 Mandell GL. Interaction of intraleukocytic bacteria and antibiotics. J Clin Invest 1973;52(7):1673-1679

90 Carryn S, Chanteux H, Seral C, Mingeot-Leclercq MP, Van Bambeke F, Tulkens PM. Intracellular pharmacodynamics of antibiotics. Infect Dis Clin North Am 2003;17(3):615-634 
91 Saginur R, Stdenis M, Ferris W, et al. Multiple combination bactericidal testing of staphylococcal biofilms from implantassociated infections. Antimicrob Agents Chemother 2006; 50(1):55-61

92 Perlroth J, Kuo M, Tan J, Bayer AS, Miller LG. Adjunctive use of rifampin for the treatment of Staphylococcus aureus infections: a systematic review of the literature. Arch Intern Med 2008;168(8): 805-819

93 Mandell GL. Uptake, transport, delivery, and intracellular activity of antimicrobial agents. Pharmacotherapy 2005;25(12, Pt 2): 130S-133S

94 Barcia-Macay M, Seral C, Mingeot-Leclercq MP, Tulkens PM, Van Bambeke F. Pharmacodynamic evaluation of the intracellular activities of antibiotics against Staphylococcus aureus in a model of THP-1 macrophages. Antimicrob Agents Chemother 2006; 50(3):841-851

95 Zinner SH, Lagast H, Klastersky J. Antistaphylococcal activity of rifampin with other antibiotics. J Infect Dis 1981;144(4):365-371

96 Norden CW, Shaffer M. Treatment of experimental chronic osteomyelitis due to staphylococcus aureus with vancomycin and rifampin. J Infect Dis 1983;147(2):352-357

97 Bayer AS, Morrison JO. Disparity between timed-kill and checkerboard methods for determination of in vitro bactericidal interactions of vancomycin plus rifampin versus methicillinsusceptible and -resistant Staphylococcus aureus. Antimicrob Agents Chemother 1984;26(2):220-223

98 Varaldo PE, Debbia E, Schito GC. In vitro activities of rifapentine and rifampin, alone and in combination with six other antibiotics, against methicillin-susceptible and methicillin-resistant staphylococci of different species. Antimicrob Agents Chemother 1985; 27(4):615-618

99 Hackbarth CJ, Chambers HF, Sande MA. Serum bactericidal activity of rifampin in combination with other antimicrobial agents against Staphylococcus aureus. Antimicrob Agents Chemother 1986;29(4):611-613

100 Walsh TJ, Auger F, Tatem BA, Hansen SL, Standiford HC. Novobiocin and rifampicin in combination against methicillin-resistant Staphylococcus aureus: an in-vitro comparison with vancomycin plus rifampicin. J Antimicrob Chemother 1986;17(1):75-82

101 Lucet JC, Herrmann M, Rohner P, Auckenthaler R, Waldvogel FA, Lew DP. Treatment of experimental foreign body infection caused by methicillin-resistant Staphylococcus aureus. Antimicrob Agents Chemother 1990;34(12):2312-2317

102 Tang HJ, Chen CC, Cheng KC, et al. In vitro efficacies and resistance profiles of rifampin-based combination regimens for biofilmembedded methicillin-resistant Staphylococcus aureus. Antimicrob Agents Chemother 2013;57(11):5717-5720

103 Niska JA, Shahbazian JH, Ramos RI, Francis KP, Bernthal NM, Miller LS. Vancomycin-rifampin combination therapy has enhanced efficacy against an experimental Staphylococcus aureus prosthetic joint infection. Antimicrob Agents Chemother 2013;57(10): 5080-5086

104 Henry NK, Rouse MS, Whitesell AL, McConnell ME, Wilson WR. Treatment of methicillin-resistant Staphylococcus aureus experimental osteomyelitis with ciprofloxacin or vancomycin alone or in combination with rifampin. Am J Med 1987;82(4A)73-75

105 Zimmerli W, Frei R, Widmer AF, Rajacic Z. Microbiological tests to predict treatment outcome in experimental device-related infections due to Staphylococcus aureus. J Antimicrob Chemother 1994;33(5):959-967

106 Chuard C, Herrmann M, Vaudaux P, Waldvogel FA, Lew DP. Successful therapy of experimental chronic foreign-body infection due to methicillin-resistant Staphylococcus aureus by antimicrobial combinations. Antimicrob Agents Chemother 1991; 35(12):2611-2616

107 Bayer AS, Lam K. Efficacy of vancomycin plus rifampin in experimental aortic-valve endocarditis due to methicillin-resistant
Staphylococcus aureus: in vitro-in vivo correlations. J Infect Dis 1985;151(1):157-165

108 Hessen MT, Pitsakis PG, Kaye D. Oral temafloxacin versus vancomycin for therapy of experimental endocarditis caused by methicillin-resistant Staphylococcus aureus. Antimicrob Agents Chemother 1990;34(6):1143-1145

109 Perdikaris G, Giamarellou H, Pefanis A, Donta I, Karayiannakos P. Vancomycin or vancomycin plus netilmicin for methicillin- and gentamicin-resistant Staphylococcus aureus aortic valve experimental endocarditis. Antimicrob Agents Chemother 1995; 39(10):2289-2294

110 Levine DP, Fromm BS, Reddy BR. Slow response to vancomycin or vancomycin plus rifampin in methicillin-resistant Staphylococcus aureus endocarditis. Ann Intern Med 1991;115(9):674-680

111 Riedel DJ, Weekes E, Forrest GN. Addition of rifampin to standard therapy for treatment of native valve infective endocarditis caused by Staphylococcus aureus. Antimicrob Agents Chemother 2008;52(7):2463-2467

112 Jung YJ, Koh Y, Hong SB, et al. Effect of vancomycin plus rifampicin in the treatment of nosocomial methicillin-resistant Staphylococcus aureus pneumonia. Crit Care Med 2010;38(1):175-180

113 Thwaites G, Auckland C, Barlow G, et al; United Kingdom Clinical Infection Research Group. Adjunctive rifampicin to reduce early mortality from Staphylococcus aureus bacteraemia (ARREST): study protocol for a randomised controlled trial. Trials 2012; 13:241

114 Auwera Van der P, Klastersky J, Thys JP, Meunier-Carpentier F, Legrand JC. Double-blind, placebo-controlled study of oxacillin combined with rifampin in the treatment of staphylococcal infections. Antimicrob Agents Chemother 1985;28(4):467-472

115 Van der Auwera P, Meunier-Carpentier F, Klastersky J. Clinical study of combination therapy with oxacillin and rifampin for staphylococcal infections. Rev Infect Dis 1983;5(Suppl 3): S515-S522

116 Johnson AP, Davies J, Guy R, et al. Mandatory surveillance of methicillin-resistant Staphylococcus aureus (MRSA) bacteraemia in England: the first 10 years. J Antimicrob Chemother 2012; 67(4):802-809

117 Peel TN, Buising KL, Dowsey MM, et al. Outcome of debridement and retention in prosthetic joint infections by methicillin-resistant staphylococci, with special reference to rifampin and fusidicacid combination therapy. Antimicrob Agents Chemother 2013;57(1):350-355

118 Aboltins CA, Page MA, Buising KL, et al. Treatment of staphylococcal prosthetic joint infections with debridement, prosthesisretention and oral rifampicin and fusidic acid. Clin Microbiol Infect 2007;13(6):586-591

119 Zimmerli W, Widmer AF, Blatter M, Frei R, Ochsner PE; ForeignBody Infection (FBI) Study Group. Role of rifampin for treatment of orthopedic implant-related staphylococcal infections: a randomized controlled trial. JAMA 1998;279(19):1537-1541

120 Senneville E, Joulie D, Legout L, et al. Outcome and predictors of treatment failure in total hip/knee prosthetic joint infections due to Staphylococcus aureus. Clin Infect Dis 2011;53(4):334-340

121 El Helou OC, Berbari EF, Lahr BD, et al. Efficacy and safety of rifampin containing regimen for staphylococcal prosthetic joint infections treated with debridement and retention. Eur J Clin Microbiol Infect Dis 2010;29(8):961-967

122 Lora-Tamayo J, Murillo O, Iribarren JA, et al; REIPI Group for the Study of Prosthetic Infection. A large multicenter study of methicillin-susceptible and methicillin-resistant Staphylococcus aureus prosthetic joint infections managed with implant retention. Clin Infect Dis 2013;56(2):182-194

123 Eisen DP, Denholm JS. Recommendations for rifampicin therapy of staphylococcal infection in Infectious Diseases Society of America prosthetic Joint Infection Guidelines are not supported by available literature. Clin Infect Dis 2013;57(1):159-160 
124 Osmon DR, Berbari EF, Berendt AR, et al; Infectious Diseases Society of America. Executive summary: diagnosis and management of prosthetic joint infection: clinical practice guidelines by the Infectious Diseases Society of America. Clin Infect Dis 2013; 56(1):1-10

125 LaPlante KL, Woodmansee S. Activities of daptomycin and vancomycin alone and in combination with rifampin and gentamicin against biofilm-forming methicillin-resistant Staphylococcus aureus isolates in an experimental model of endocarditis. Antimicrob Agents Chemother 2009;53(9):3880-3886

126 Miró JM, García-de-la-Mària C, Armero Y, et al; Hospital Clinic Experimental Endocarditis Study Group. Addition of gentamicin or rifampin does not enhance the effectiveness of daptomycin in treatment of experimental endocarditis due to methicillin-resistant Staphylococcus aureus. Antimicrob Agents Chemother 2009; 53(10):4172-4177

127 Rose WE, Berti AD, Hatch JB, Maki DG. Relationship of in vitro synergy and treatment outcome with daptomycin plus rifampin in patients with invasive methicillin-resistant Staphylococcus aureus infections. Antimicrob Agents Chemother 2013;57(7):3450-3452

128 Jugun K, Vaudaux P, Garbino J, et al. The safety and efficacy of high-dose daptomycin combined with rifampicin for the treatment of Gram-positive osteoarticular infections. Int Orthop 2013;37(7):1375-1380

129 Lora-Tamayo J, Parra-Ruiz J, Rodríguez-Pardo D, et al. High doses of daptomycin $(10 \mathrm{mg} / \mathrm{kg} / \mathrm{d})$ plus rifampin for the treatment of staphylococcal prosthetic joint infection managed with implant retention: a comparative study. Diagn Microbiol Infect Dis 2014; 80(1):66-71

130 Watanakunakorm C, Glotzbecker C. Enhancement of the effects of anti-staphylococcal antibiotics by aminoglycosides. Antimicrob Agents Chemother 1974;6(6):802-806

131 Houlihan HH, Mercier RC, Rybak MJ. Pharmacodynamics of vancomycin alone and in combination with gentamicin at various dosing intervals against methicillin-resistant Staphylococcus aureus-infected fibrin-platelet clots in an in vitro infection model. Antimicrob Agents Chemother 1997;41(11):2497-2501

132 Lemonovich TL, Haynes K, Lautenbach E, Amorosa VK. Combination therapy with an aminoglycoside for Staphylococcus aureus endocarditis and/or persistent bacteremia is associated with a decreased rate of recurrent bacteremia: a cohort study. Infection 2011;39(6):549-554

133 Cosgrove SE, Vigliani GA, Fowler VG Jr, et al. Initial low-dose gentamicin for Staphylococcus aureus bacteremia and endocarditis is nephrotoxic. Clin Infect Dis 2009;48(6):713-721

134 Baltch AL, Ritz WJ, Bopp LH, Michelsen PB, Smith RP. Antimicrobial activities of daptomycin, vancomycin, and oxacillin in human monocytes and of daptomycin in combination with gentamicin and/or rifampin in human monocytes and in broth against Staphylococcus aureus. Antimicrob Agents Chemother 2007; 51(4):1559-1562

135 Credito K, Lin G, Appelbaum PC. Activity of daptomycin alone and in combination with rifampin and gentamicin against Staphylococcus aureus assessed by time-kill methodology. Antimicrob Agents Chemother 2007;51(4):1504-1507

136 Debbia E, Pesce A, Schito GC. In vitro activity of LY146032 alone and in combination with other antibiotics against gram-positive bacteria. Antimicrob Agents Chemother 1988;32(2):279-281

137 LaPlante KL, Rybak MJ. Impact of high-inoculum Staphylococcus aureus on the activities of nafcillin, vancomycin, linezolid, and daptomycin, alone and in combination with gentamicin, in an in vitro pharmacodynamic model. Antimicrob Agents Chemother 2004;48(12):4665-4672

138 DeRyke CA, Sutherland C, Zhang B, Nicolau DP, Kuti JL. Serum bactericidal activities of high-dose daptomycin with and without coadministration of gentamicin against isolates of Staphylococ- cus aureus and Enterococcus species. Antimicrob Agents Chemother 2006;50(11):3529-3534

139 Rose WE, Leonard SN, Rybak MJ. Evaluation of daptomycin pharmacodynamics and resistance at various dosage regimens against Staphylococcus aureus isolates with reduced susceptibilities to daptomycin in an in vitro pharmacodynamic model with simulated endocardial vegetations. Antimicrob Agents Chemother 2008;52(9):3061-3067

140 Baltch AL, Ritz WJ, Bopp LH, Michelsen P, Smith RP. Activities of daptomycin and comparative antimicrobials, singly and in combination, against extracellular and intracellular Staphylococcus aureus and its stable small-colony variant in human monocytederived macrophages and in broth. Antimicrob Agents Chemother 2008;52(5):1829-1833

141 Kullar R, Casapao AM, Davis SL, et al. A multicentre evaluation of the effectiveness and safety of high-dose daptomycin for the treatment of infective endocarditis. J Antimicrob Chemother 2013;68(12):2921-2926

142 Steed ME, Werth BJ, Ireland CE, Rybak MJ. Evaluation of the novel combination of high-dose daptomycin plus trimethoprim-sulfamethoxazole against daptomycin-nonsusceptible methicillin-resistant Staphylococcus aureus using an in vitro pharmacokinetic/pharmacodynamic model of simulated endocardial vegetations. Antimicrob Agents Chemother 2012; 56(11):5709-5714

143 Avery LM, Steed ME, Woodruff AE, Hasan M, Rybak MJ. Daptomycin-nonsusceptible vancomycin-intermediate staphylococcus aureus vertebral osteomyelitis cases complicated by bacteremia treated with high-dose daptomycin and trimethoprim-sulfamethoxazole. Antimicrob Agents Chemother 2012;56(11): 5990-5993

144 Di Carlo P, D’Alessandro N, Guadagnino G, et al. High dose of trimethoprim-sulfamethoxazole and daptomycin as a therapeutic option for MRSA endocarditis with large vegetation complicated by embolic stroke: a case report and literature review. Infez Med 2013;21(1):45-49

145 Dumitrescu O, Badiou C, Bes M, et al. Effect of antibiotics, alone and in combination, on Panton-Valentine leukocidin production by a Staphylococcus aureus reference strain. Clin Microbiol Infect 2008;14(4):384-388

146 Dumitrescu O, Boisset S, Badiou C, et al. Effect of antibiotics on Staphylococcus aureus producing Panton-Valentine leukocidin. Antimicrob Agents Chemother 2007;51(4): 1515-1519

147 Diep BA, Afasizheva A, Le HN, et al. Effects of linezolid on suppressing in vivo production of staphylococcal toxins and improving survival outcomes in a rabbit model of methicillinresistant Staphylococcus aureus necrotizing pneumonia. J Infect Dis 2013;208(1):75-82

148 Rouzic N, Janvier F, Libert N, et al. Prompt and successful toxintargeting treatment of three patients with necrotizing pneumonia due to Staphylococcus aureus strains carrying the PantonValentine leukocidin genes. J Clin Microbiol 2010;48(5): 1952-1955

149 Li HT, Zhang TT, Huang J, Zhou YQ Zhu JX, Wu BQ. Factors associated with the outcome of life-threatening necrotizing pneumonia due to community-acquired Staphylococcus aureus in adult and adolescent patients. Respiration 2011;81(6): 448-460

150 Subedi S, Baird R, Tong SY. Does the Addition of Lincosamides Have Mortality Benefit in Severe Staphylococcal Infection? C-765. In: Interscience Conference on Antimicrobial Agents and Chemotherapy; 2014; Washington, DC; 2014

151 Gillet Y, Dumitrescu O, Tristan A, et al. Pragmatic management of Panton-Valentine leukocidin-associated staphylococcal diseases. Int J Antimicrob Agents 2011;38(6):457-464 


\section{Combination Antibiotic Treatment of Serious MRSA Infections Davis et al.}

152 Health Protection Agency. Guidance on the Diagnosis and Management of PVL-Associated Staphylococcus aureus Infections (PVL-SA) in England, 2nd ed. London, UK: HPA; 2008

153 Lin G, Pankuch G, Appelbaum PC, Kosowska-Shick K. Antistaphylococcal activity of oritavancin and its synergistic effect in combination with other antimicrobial agents. Antimicrob Agents Chemother 2014;58(10):6251-6254
154 Lin G, Pankuch GA, Ednie LM, Appelbaum PC. Antistaphylococcal activities of telavancin tested alone and in combination by timekill assay. Antimicrob Agents Chemother 2010;54(5):2201-2205

155 Xu-hong Y, Falagas ME, Dong W, Karageorgopoulos DE, De-feng L, Rui W. In vitro activity of fosfomycin in combination with linezolid against clinical isolates of methicillin-resistant Staphylococcus aureus. J Antibiot (Tokyo) 2014;67(5):369-371 